\title{
Electric tracing system temperature control of submarine heavy oil pipeline based on genetic algorithm
}

\author{
Li Ding and Jiasheng Zhang ${ }^{\mathrm{a}}$ \\ China university of Petroleum, College of Information and Control Engineering, 266580, Qingdao Shandong, China
}

\begin{abstract}
In order to optimize the heating dynamic response of heavy oil in the submarine pipeline, the control system of the pipeline electric current tracing system is designed. Firstly, the mathematical model of electric current tracing system for heavy oil pipeline is established based on mechanism modeling method. Aims at the controlled object with First Order Plus Dead Time (FOPDT) model, temperature is controlled with the most widely used PID controller in industrial process control. Secondly, a variety of tuning methods for controller parameters are discussed. Finally, the temperature control method based on genetic algorithm tuning is established, and comparisons are drawn by MATLAB simulation. The results show that the temperature overshoot of PID temperature control method based on genetic algorithm is smaller, and the time for heating to target temperature is also significantly reduced. Therefore, PID temperature control method based on genetic algorithm can shorten the tuning time for parameters significantly, it has the higher heating efficiency with less energy cost, furthermore, and it can well meet the needs of heating system control.
\end{abstract}

Keywords: submarine heavy oil pipeline; FOPDT; genetic algorithm; temperature control.

\section{Introduction}

Heavy oil is an important component in petroleum hydrocarbons energy, and its rheological properties vary greatly according to temperature. The lower the temperature, the higher the viscosity, which is not conducive to onshore heavy oil pipeline transportation. The transportation method of heating and reducing viscosity is adopted in the onshore heavy oil pipeline, which can improve the flow temperature of heavy oil and reduce the viscosity of heavy oil, with the aim of improving the mobility of heavy oil and reducing the friction loss in the pipeline. Marine heavy oil reserves of China are abundant, but under the condition of offshore platform, the influence of the submarine pipeline at the entrance of the engineering facilities, the thermal load limit and the complex working conditions of the seabed, and the operating economy and security of submarine heavy oil pipeline is greatly deteriorated than onshore. The efficiency and security transportation technology of heavy oil pipeline are becoming a bottleneck restricting of the development of marine oil resources. At this time, the submarine pipeline electric current tracing technology came into being, which refers to the use of electrical energy is added to the heated objects to replenish the heat loss in the transport process, and the flow medium temperature maintain within a certain temperature range. Electric current tracing can be divided into electric heating cable, electromagnetic induction heating, direct electric heating and

a Corresponding author : zjsycy2003@126.com. 
other methods. Practice shows that due to the high power and long distance need of submarine pipeline, and the underwater part cannot set electrical junction box, combined with above, skin electric current tracing (SECT) [1] is considered.

Although the heating process keeps improving with the development of power electronic devices, the research on the temperature control algorithm of submarine oil pipeline is quite few. The research of submarine pipeline electric current tracing system, which has a controlled object with first order plus time delay (FOPDT), the most widely used PID controller of industrial process control in temperature control should be used. This paper compares among varieties of PID controller parameter tuning methods: PID tuning based on Ziegler-Nichols method [2], optimal control PID tuning based on the optimal ISTE index [3], optimal control PID tuning based on modified ITAE index [4], after defining the scope of the PID controller parameters tuning, using the global optimization ability of genetic algorithm, the parameters of the PID are determined more precisely [5]. Finally, the PID control method based on genetic algorithm and the traditional method are analyzed and contrasted.

\section{Skin electric current tracing system for submarine pipeline}

Skin electric current tracing (SECT) system is based on skin effect and proximity effect principle. An SECT heating system is as shown in Figure 1, the device consists of carbon steel tube as heat pipe and SECT cable in the heat pipe [6]. One end of AC power source is connected with the outer wall of SECT tube. And the other end is transmitted to inner SECT tube with a special single core cable and attached to the tube wall at the end.

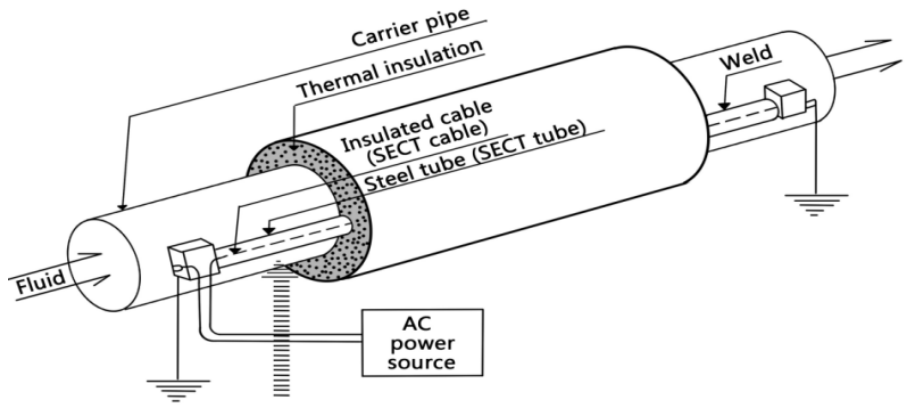

Figure 1. An SECT system.

After powered on, the charges flow through the insulated cable and the heat pipe will produce skin effect and proximity effect, due to the proximity effect, the current trends to flow to the outer wall of cable conductor and inner wall of SECT tube, because of the steel tube has a high relative permeability, the system shows a strong skin effect. The current flowing through the inner wall of SECT tube is limited in a thin layer, and the thin layer generates enough Joule heat to meet the heating needs. The outer wall of the tube has no current, and it proves the SECT system has the natural insulation structures.

\section{The mathematical model of SECT system of submarine pipeline}

For the electric current tracing in oil pipeline, the steel tube welded to the pipeline, the heat-resistant cable passes through the steel tube, thus a closed loop is formed. Then AC is powered on, the steel tube and heat-resistant cable generate Joule heat due to the AC impedance, and then the temperature of heavy oil pipeline rises. On the basis of heat transfer principle, the heat transfer equation is obtained. This paper discussed steady heat transfer process that is the heat transfer process in which the temperature of each point in one object does not change over time. Assumptions:

(1) Ambient temperature does not change due to the temperature change while heating;

(2) The heat transfers from the heating unit to the heated unit straight-line, and no energy loss while transferring. 
According to the law of conservation of energy, the heat balance equation is obtained, the heat absorbed by pipeline is equal to the difference of heat generates by SECT tube and the heat loss of pipeline to the surrounding environment [7], i.e.

$$
G C \frac{d T}{d t}=Q_{i}-Q_{o u t}
$$

Where $G$ is the quality of heavy oil in the pipeline, $C$ is the specific heat of heavy oil, $T$ is the working temperature of the pipeline, $Q_{i}$ is the heat generates by SECT tube in unit time, $Q_{\text {out }}$ is the loss of heat from pipeline to the surrounding environment in unit time. The heat from pipeline to the surrounding environment in unit time is:

$$
Q_{\text {out }}=K \pi D L\left(T-T_{0}\right)
$$

Where $K$ is the overall heat transfer coefficient from heavy oil flow to the surrounding agent $\left(W /\left(m^{2} \cdot K\right)\right), T_{0}$ is the external ambient temperature $\left({ }^{\circ} \mathrm{C}\right), D$ is the diameter of pipeline (mm), $L$ is the length of pipeline (m).

Due to the heat generated by the electric tracing $Q_{i}$ is proportionate to the square of applied voltage in unit time, $Q_{i}$ and $U$ are nonlinearly related, which can be linearized $K_{\mu}=\frac{\Delta Q_{i}}{\Delta U}$ near the equilibrium point $\left(Q_{0}, U_{0}\right)$ :

$$
G C \frac{d(\Delta T)}{d t}=K_{\mu} \Delta U-K \pi D L(\Delta T)
$$

For further simplify

$$
\tau \frac{d(\Delta T)}{d t}+\Delta T=K_{1} \Delta U
$$

Where

$$
\tau=\frac{G C}{K \pi D L}
$$

is the time inertia constant of pipeline;

$$
K_{1}=\frac{K_{\mu}}{K \pi D L}
$$

is the amplification factor of the pipeline.

Under the zero initial condition, take up the Laplace transform of above and obtained:

$$
G(s)=\frac{\Delta T(s)}{\Delta U}=\frac{K_{1}}{1+\tau s}
$$

In consideration of the hysteresis quality of the system, a delay link is added in the system and the transfer function is as following:

$$
G(s)=\frac{K_{1}}{1+\tau s} e^{-\tau_{0} s}
$$


Written in general

$$
G(s)=\frac{K_{p}}{1+T_{p} s} e^{-\tau s}
$$

It can be seen that the load of the electric tracing system can be regarded as a first order plus dead time (FOPDT).

For a pipeline with the diameter of $\Phi_{325}$, oil transportation capacity of $57 \mathrm{Kg} / \mathrm{s}$, length of $1000 \mathrm{~m}$, the corresponding parameters are substituted into the above formula (3-5) and (3-6), it can generally obtained $K_{p}=0.27, T_{p}=4800, \tau=300$. And the system transfer function is $G(s)=\frac{0.27 e^{-300 s}}{4800 s+1}$.

\section{The design of the electric current tracing system control method for the submarine pipeline}

SECT system is a controlled object with time-variant, nonlinear and large inertia, which caused the controlled variable cannot reflect the withstand disturbance of systems timely, and it leads to a greater overshoot and stability of the control system get lower. The adjusting time is lengthened, and even shock, divergence occurs, makes the dynamic quality of the system significantly worse, which resulting in more difficult for system design and control. In order to ensure the constant temperature of crude oil in pipeline transportation, in the SECT system, the input variable of temperature controller is temperature deviation, the executive component of the system is variable frequency power supply, the larger the ratio, the greater the output power, therefore the control variable output of controller is regarded as the input of PWM frequency power ratio, and the whole system can realize the control of temperature, the temperature control structure diagram is shown in Figure 2.

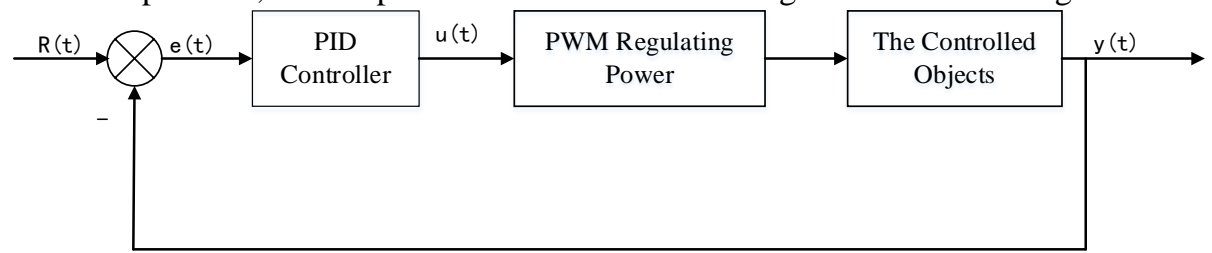

Figure 2.Structure diagram of temperature control.

\subsection{PID controller parameters optimization and effect}

The design of PID controller [8] is the design of the three important parameters. According to the FOPDT model related to PID controller design problems for this kind of controlled object, in 1942, the empirical formula proposed by Nichols and Ziegler has the greatest impact, so that in many projects it is still in use. In addition, there are many other setting methods, nowadays the ISTE optimal formula proposed by Zhuang Minxia is recognized as a better PID tuning methods, but the scope of the algorithm is $0.1 \leq L / T \leq 2$, it is not suitable for large dead time control system, which has a certain limitation [9]. In order to overcome the defects, Zhang Fubo put forward the optimal setting formula based on the PID controlled object characteristic parameters [4], that is the ITAE setting method,

$$
\left.\begin{array}{c}
K_{p}=1.034 K^{-1} T^{0.8733} L^{-0.8823} \\
T_{1}=1.6013 T^{0.9011} L^{0.0844} \\
T_{D}=0.3088 T^{0.1131} L^{0.9047}
\end{array}\right\}
$$


This algorithm expanded the applicable scope to $0.02 \leq L / T \leq 4$, which shows a better control effect. Selecting the characteristic parameters of pipe electric current tracing system, and respectively according to Ziegler Nichols setting method, ISTE optimal setting method and the ITAE setting method to obtain controller parameters are listed in Table 1.

Table 1. Comparison of PID controller parameters under different setting methods

\begin{tabular}{ccccccccccccc}
\hline \multicolumn{3}{c}{ parameters } & \multicolumn{1}{c}{ Ziegler-Nichols } & \multicolumn{4}{c}{ Zhuang \& Atherton ISTE } & \multicolumn{3}{c}{ Zhang et al ITAE } \\
$\mathrm{K}$ & $\mathrm{T}$ & $\mathrm{L}$ & $K_{P}$ & $T_{I}$ & $T_{D}$ & $K_{P}$ & $T_{I}$ & $T_{D}$ & $K_{P}$ & $T_{I}$ & $T_{D}$ \\
& & & & & & & & & & & & \\
0.27 & 4800 & 300 & 71.11 & 660 & 150 & 21.06 & 330.3 & 180.02 & 40.9 & 5503.2 & 140.31 \\
\hline
\end{tabular}

Editing $\mathrm{M}$ files for simulation analysis under the MATLAB environment, with the system target temperature of $80^{\circ} \mathrm{C}$, simulation results are shown in Figure 3.

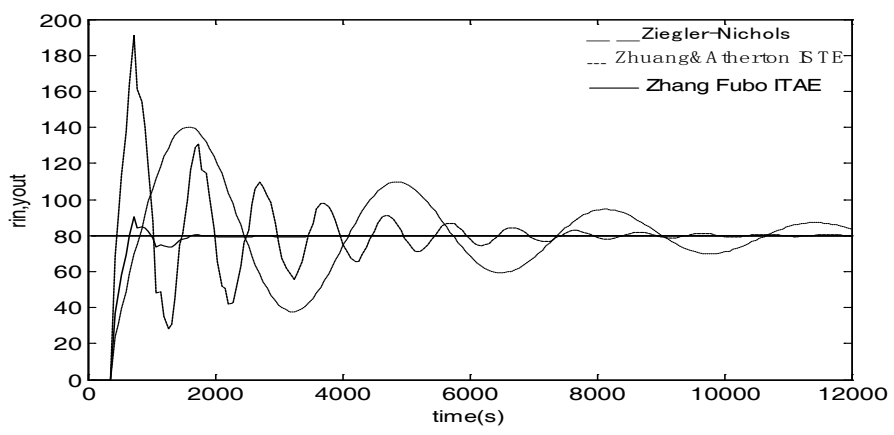

Figure 3.Comparison of PID simulation results of SECT system.

It can be seen from the figure that setting method based on Ziegler-Nichols and optimal setting method based on ISTE are not ideal for either, which appearing large fluctuations and instability of the system. And optimal setting method based on ITAE reaches stability in 2000s, with the system adjusting time greatly shortened and achieved PID tuning requirements. Based on the results above, PID temperature controller tuning parameters are preliminarily selected for SECT system as $K_{P}=40.96, K_{I}=0.00744, K_{D}=5746.95$.

\subsection{Optimal setting of PID control parameters based on genetic algorithm}

Genetic algorithm is a kind of adaptive global optimization probability search method which simulates the biological genetic and evolutionary processes formed in the natural environment.

In order to achieve good control effect in PID control, the best combination of proportion, integral and differential control is needed to achieve the win-win purpose [10]. Genetic algorithm does not require any initial information and can find the global optimal solution of optimization algorithm, so it is entirely possible for using genetic algorithm to optimize three parameters in PID controller [11].

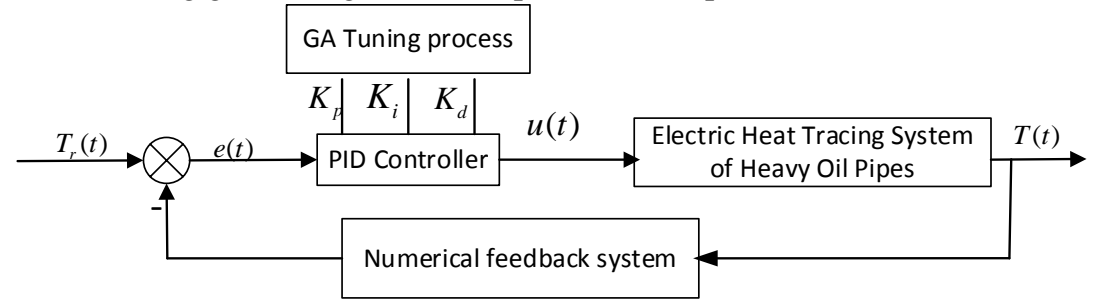

Figure 4. Schematic diagram of electric tracing system based on genetic algorithm 
Using genetic algorithm to optimize the three parameters $K_{P} K_{I} K_{D}$, the specific steps are as following:

(1) Determine the approximate range of the three parameters and the length of the encoding, then begin to encode;

(2) Randomly generates $n$ individuals to constitute the initial population;

(3) Determine the individual evaluation method that is to calculate the adaptive value of the individual in the population after decoding.

In this paper, the design of the objective function consists of three parts: error, control variable, rise time. In order to obtain satisfying dynamic characteristics on transition, the error absolute value time integral performance index is selected as the minimum objective function of parameter selecting. In order to avoid the overshoot, the overshoot is added to the performance index, as one part of the optimal performance index. In order to prevent excessive control variable, the square of control variables is added to the performance index, so in this paper, the objective function is designed as:

$$
\left\{\begin{array}{lr}
J=\int_{0}^{\infty}\left(w_{1}|e(t)|+w_{2}|e y(t)|+w_{3} u^{2}(t)\right) d t+w_{4} \cdot t_{u} & \text { ey }(t)<0 \\
J=\int_{0}^{\infty}\left(w_{1}|e(t)|+w_{3} u^{2}(t)\right) d t+w_{4} \cdot t_{u} & \text { others }
\end{array}\right.
$$

In this formula, $e(t)$ is the error of system, $e y(t)=y(t)-y(t-1)$ is overshoot, $y(t)$ is the output of the controlled object, $u(t)$ is the control input quantity, $t_{u}$ is the rise time, $w_{1}, w_{2}, w_{3}, w_{4}$ are the weights and $w_{2} \gg w_{1}$, the adaptive function can be obtained as $f=1 / \mathrm{J}$.

(4) Design of genetic operators;

By applying the proportional selection operator, the single point crossover operator and the basic bit mutation operator, the population is operated, and the new species is produced.

(5) Determine the operating parameters of the genetic algorithm;

(6) Repeat steps (3) and (4), until the parameters converge or achieve a predetermined index.

The solution of genetic algorithm is shown in Figure 5.

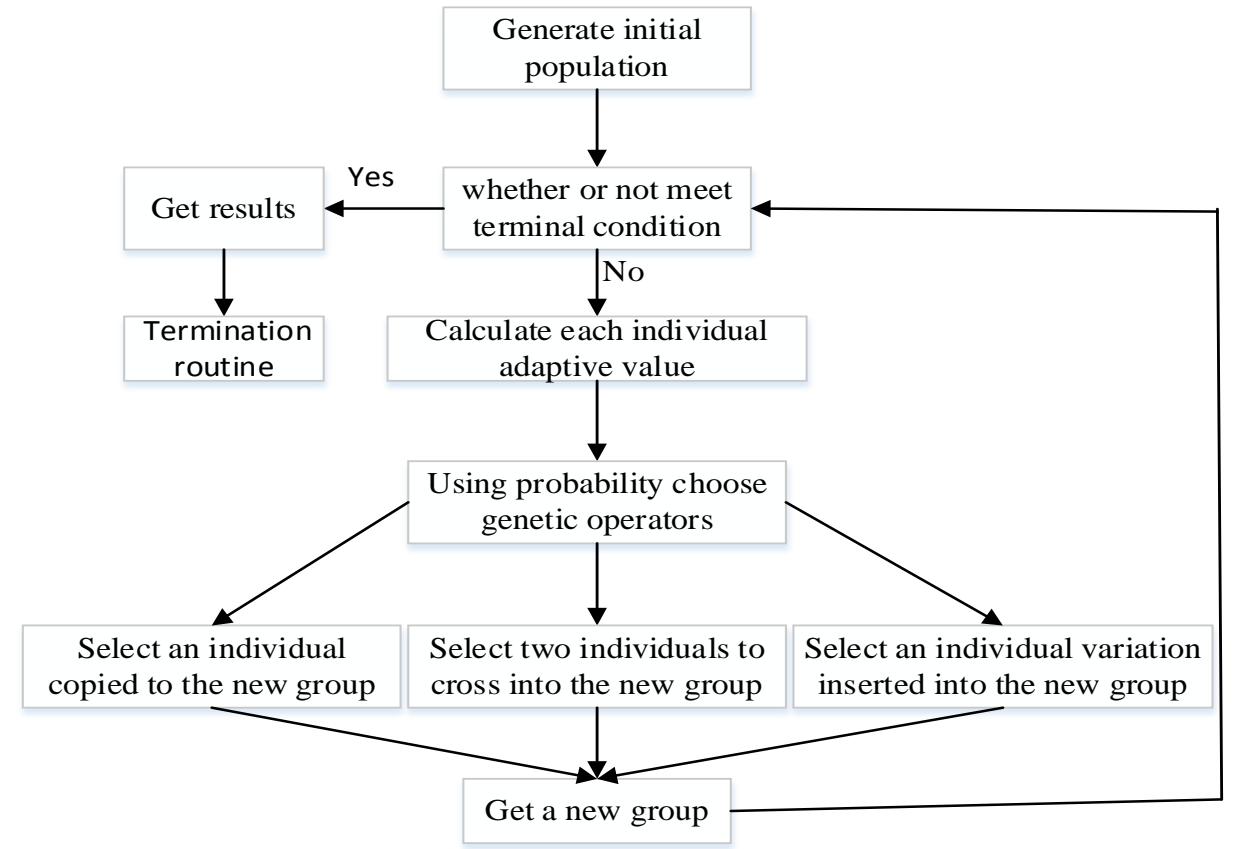

Figure 5. Flow chart of basic genetic algorithm. 
Simulation experiment studies of PID control parameters based on genetic algorithm carry out the MATLAB simulation which aims at the submarine pipeline SECT system $G(s)=0.27 /(4800 s+1) e^{-300 s}$.

The initial parameter settings: population size $M=30$, termination evolution algebra $G=100$, probability of crossover $P_{c}=0.9$, adaptive mutation probability method, the adaptive value is inversely proportional to the mutation probability. In this paper, Section 4.1 has tuning value of each parameter, so the range of parameter $K_{P}$ is [0,40], the range of $K_{I}$ is [0,0.1], the range of $K_{D}$ is [0,5000] and $w_{1}=0.999, w_{2}=100, w_{3}=0.001, w_{4}=2.0$. Using real number encoding method, after 100 generations of evolution, the optimized PID parameters are as follows: $K_{P}=24.33$, $K_{D}=876.48, K_{I}=0.005$, performance index $J=36.15$. The optimization process of cost function $J$ is shown in Figure 6.

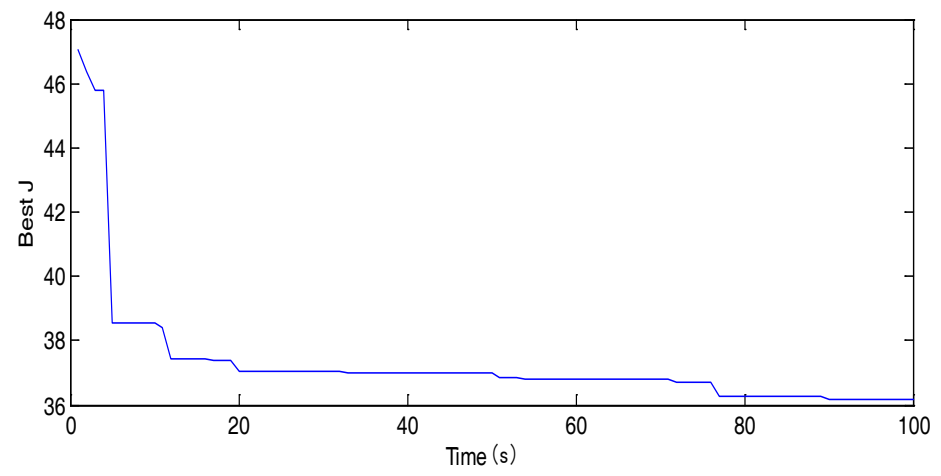

Figure 6. Optimization process of cost function $J$.

The step response curve of the system is shown in Figure 7.

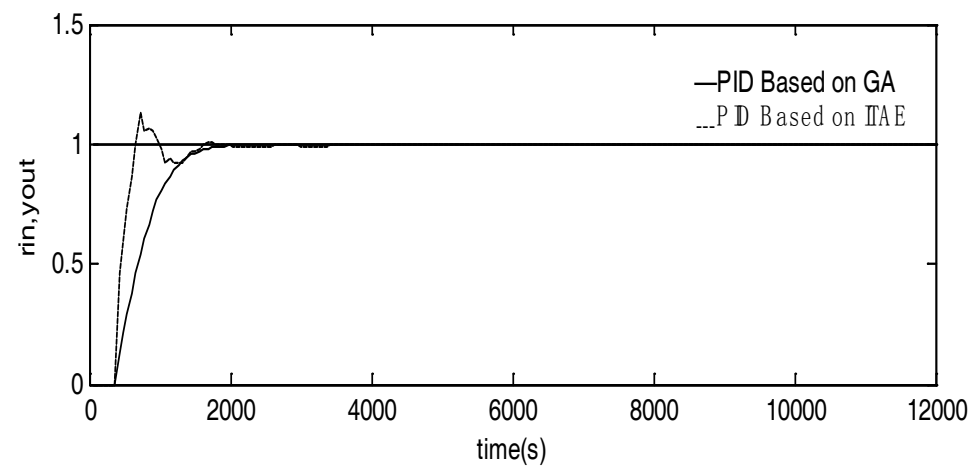

Figure 7. Step response curve of PID before and after tuning.

It can be seen from the figure above that rise time $t_{r}=1350 \mathrm{~s}$, the system overshoot $\sigma \%=0.95 \%$, compares with the ITAE index has been greatly improved. In order to verify the anti-interference ability of the system, an interference of 10 is added to controller output at the 50th sampling time (3000s), the obtained response results is shown in Figure 8. The PID parameters after optimization: $K_{P}=23.204, K_{D}=292.9861, K_{I}=0.005$, performance index $J=35.8896$. 


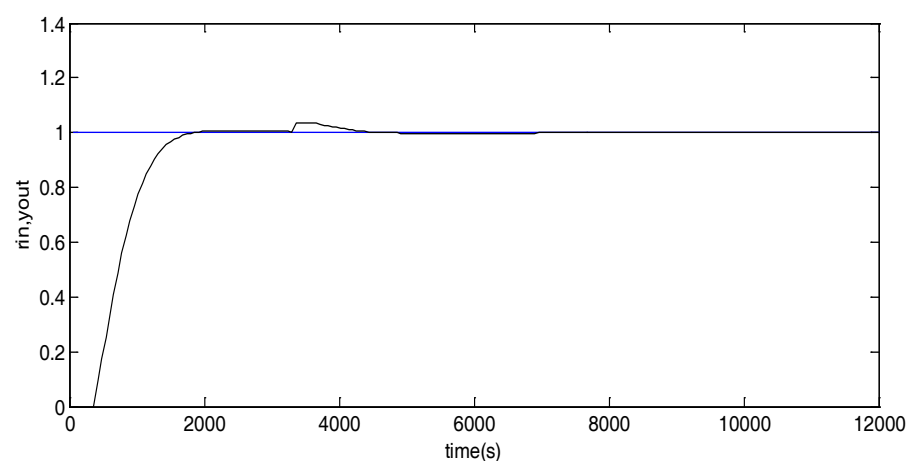

Figure 8. Characteristic curve after adding an interference.

The simulation analysis shows that the genetic algorithm optimized PID control improves in precision and anti-interference ability, and the control effect achieve the requirements.

\section{Conclusion}

(1) PID control is widely used in industrial process control of the control algorithm. While designing, in order to avoid repeated trial to get the optimal combination of the three parameters proportional, integral and differential, many scholars proposed the effective PID parameter tuning method. This paper according to the object of SECT system, compares based on the Ziegler-Nichols PID tuning method, optimal control PID tuning based on ISTE index and to achieve the optimal tuning results;

(2) On the optimal results basis of optimal control PID tuning based on the improved ITAE, using genetic algorithm to optimize tuning and get optimal PID tuning parameters, in order to verify its effectiveness and anti-interference, the carried out MATLAB simulation experiments proves the rapidity, accuracy and stability of the system have been improved obviously.

Due to the algorithm has a certain universality, this PID control based on genetic algorithm in SECT system can be extended to first order plus dead time model (FOPDT), intelligent PID control algorithm can be applied in more fields, and realized the digitization and intelligence in oilfield as soon as possible.

\section{Acknowledgement}

This study was financially supported by "the Fundamental Research Funds for the Central Universities"(No.16CX06051A).

\section{Reference}

1. H. Takaki, A. Iwasaki,S. Oiwa and J. Kurano. ISOPE, 184, (1993)

2. Ziegler JG, Nichols NB. ASME, 64, 759 768, (1944)

3. Zhuang M, Atherton D P. IEE, Part D, 140, 216 224, (1993)

4. F. B. Zhang, G.D. Wang, D.H. Zhang, X.H. Liu. J NORTHEAST UNIV, 26,754 757, (2005)

5. Alberto Leva, Martina Maggio. 49th IEEE CDC, 3319, (2010)

6. G.F. Liu, X. Li, C. Wei. Automation Application, 6, 3 4, (2015)

7. Z.K. Yin. Zhejiang Univ. (2003)

8. R.D. Kokate, L.M. Waghmare, S.D. Deshmukh. ARTCom, 71 75, (2010)

9. D.Y. Xue. Computer Aided Control Systems Design Using MATLAB Language (Third Edition). (2012)

10. A.S. Ruben, H.G. Oscar, CCE, 1 6, (2015)

11. D. Pradeep Kannan, S. Sathiyamoorthy. ICACCCT, 130 135, (2014) 